\section{A systematic procedure for the elasto-dynamic modeling and identification of robot manipulators}

\author{
Mathieu Rognant, Eric Courteille, and Patrick Maurine
}

\begin{abstract}
This paper presents a systematic procedure for the elastodynamic modeling of industrial robots, applicable either to serial or parallel manipulators. This procedure is based on a 3D space generalization of the Equivalent Rigid Link System (ERLS) description, the Finite Element Method (FEM) and the Lagrange principle. It considers flexible links and joints, and leads to generic equations of motion expressed according to the angles of the actuated joints and the independent elastic degrees of freedom. An efficient identification process through modal analysis is detailed, and the description of damping and joint behavior according to the model application is discussed. The method is applied to a 3D Delta-like parallel structure and successfully validated through an experimental impact testing-based modal analysis.
\end{abstract}

Index Terms-Elasto-dynamic Modeling, Calibration and Identification, Mechanism Design, Experimental Modal Analysis.

\section{INTRODUCTION}

In recent decades, much research has focussed on dynamic modeling of robot manipulators through the definition of accurate mathematical models, which are used to predict their dynamic behavior. However, the assumption of rigid body is no longer sufficient if high speed, high load and high accuracy applications are considered: lasercutting or machining, for instance. Therefore, flexibilities induced by the different elements of the manipulator structure, such as links, joints, base and tools, have to be considered carefully in the modeling. In that research, significant accomplishments have been made in the kinematic description of flexible multibody systems and have been applied to manipulators. The first dynamic modeling studies have concerned serial manipulators [1]-[4] and more recently Parallel Kinematic Machines (PKMs) [5], [6]. These models are useful for the optimization of robot design [7], [8] and to implement active vibration control for real-time applications [9].

In order to implement these models in an industrial context, we propose extending existing system modeling and identification techniques to form a general tool for characterizing the elastodynamic behavior of 3D serial and parallel manipulators.

The paper is organized as follows. In the first section, the various previously published modeling principles are reviewed and analyzed. Following that, the proposed elasto-dynamic modeling procedure and its systematic formulation are presented. Thereafter, its implementation and identification according to the applications concerned are discussed. The procedure is then applied to a 3D, Delta-like, parallel structure in order to verify its efficiency through experimental correlation. The valid domain of the model is limited to smaller deflections. Indeed, modal identification was conducted using an instrumented impact hammer. This technique essentially results in a linear interpretation of the system in a particular configuration, but the methodology establishes a solid basis for future research on large nonlinear deflections of these structures or for the development of control algorithms.

\section{Modeling}

\section{A. Modeling principle}

In the literature, elasto-dynamic models are commonly based upon the following techniques.

Mathieu Rognant, Eric Courteille, and Patrick Maurine are with the Université Européenne de Bretagne, INSA-LGCGM - EA 3913, 20, avenue des Buttes de Coesmes 35043 RENNES Cedex, France (e-mail: mathieu.rognant@onera.fr; eric.courteille@insa-rennes.fr; patrick.maurine@insa-rennes.fr).
1) Floating reference frame: In this method, the body motion, assuming small deformations, is considered to be the superposition of a non-linear rigid body displacement and a linear elastic displacement. As a result two sets of coordinates are used to describe the configuration of the deformable bodies; one set describes the location and orientation of a selected body coordinate system while the second set describes the elastic displacement of the body with respect to its coordinate system. The first set of coordinate systems is obtained using the classic rigid method of description as in Newton-Euler [10], [11] or $(4 \times 4)$ transformation matrices [1], [12], [13]. In particular, Chang and Hamilton in [12] put forward a kinematic description of flexible-link manipulators using the ERLS Model. Elastic behavior is implemented by using the Assumed Modes Method (AMM) [1], [4], [13], [14], or FEM [5], [15]-[18] in order to obtain a finitedimensional model.

2) Lumped parameter model: Lumped parameter models describe manipulators as a set of rigid bodies which are connected by springs and/or dampers. These springs and/or dampers are used to describe the viscoelastic behavior of the joints and links. This method has the advantage that rigid body methodologies can be used [19]. However, the accuracy and consistency of the model obtained depend on the number, size and location of the rigid segments used.

3) Convected coordinate system and large rotation vectors: The convected coordinate system is employed using the incremental approach, which is used to solve large rotation problems in nonlinear finite element analysis. In this approach, the kinematic equations are first defined in the element coordinate system. It is assumed that the rotation of the element between two successive configurations is small enough that the use of the nodal coordinates to describe the rotation can be justified. However, Shabana demonstrates in [20] that the incremental finite element formulation does not lead to an exact modeling of rigid-body dynamics for large-rotation motion of the structures. In order to avoid this problem, Simo introduces in [21], [22] the large rotation vector formulation but this formulation can lead to singularity problems [23].

4) Absolute nodal coordinate: Absolute Nodal Coordinate Formulation (ANCF) is a recently developed non-incremental finite element approach that has been specially designed for large-deformation, multibody applications [24], [25]. Nodal coordinates are the position vector of the nodal points with respect to the global coordinate system and the vector of the displacement gradients, also defined in the global coordinate system. The ANCF is equivalent to the floating reference frame formulation but leads to a different structure for the dynamic equations of motion. In the ANCF the mass matrix is constant, Centrifugal and Coriolis inertia forces are equal to zero and the stiffness matrix is non linear even in the case of linear elastic behavior [26]. Many simulations based on this formulation have been recently presented [27], [28].

In recent research, one can see that the floating reference frame description and FEM are applied to planar PKMs [5], [17]. The accuracy of this approach is experimentally demonstrated in [17] and its relevance for real-time applications is shown in [5] by Wang and Mills. The comparison of the AMM and FEM approaches, realized in [29] by Theodore and Ghosal, shows that FEM is recommended for flexible multilink manipulators and it is ideally suited for dynamic, model-based, real-time controller implementation. In order to simplify the application of elasto-dynamic modeling in the industrial context, we suggest synthesizing these works in a systematic procedure based on FEM, a 3D space generalization of the ERLS description and the Lagrange principle.

The main advantages of our work resides in the following points. Firstly, the elasto-dynamic model can be derived automatically. This is done using a simple description of the structure through 
the establishment of two tables giving respectively the values of the geometrical and elastic parameters related to all bodies of the structure. Secondly, the configuration of the manipulator is described using the independent elastic DOF expressed in the global frame and the values of the actuated joints. These values can be easily measured, which simplifies the identification process. This method allows the description of the behavior of the joints using an appropriately located stiffness parametrization. Finally, as the elastic potential energy of the joints is modeled, the constraint equations which link the elastic DOF are simplified. Thus, generic expressions of stiffness and mass matrices as a function of the independent coordinates are obtained which leads to a simple modal analysis computation.

\section{B. Kinematic description}

1) ERLS description: In order to achieve a systematic procedure, Khalil and Kleinfinger's notation [30] is used to perform the ERLS description. This method is derived from the well-known Denavit and Hartenberg's notation and can be used to describe with a minimum set of parameters the open- and closed-loop robots. This notation is based on a specific frame definition, detailed in [30] and [31]. On each body $C_{i}$, a frame $R_{i}$ is assigned for which, $\mathbf{z}_{i}$ axis is aligned with the axis of joint $\mathrm{i}$ and $\mathbf{x}_{i}$ is along the common normal to $\mathbf{z}_{j}$. Therefore, the transformation from $R_{i}$ with respect to the previous frame $R_{j}$ is defined by the $(4 \times 4)$ matrix ${ }^{j} \mathbf{T}_{i}$ :

$$
\begin{array}{r}
{ }^{j} \mathbf{T}_{i}=\operatorname{rot}\left(\mathbf{z}_{j}, \gamma_{i}\right) \operatorname{trans}\left(\mathbf{z}_{j}, b_{i}\right) \operatorname{rot}\left(\mathbf{x}_{j}, \alpha_{i}\right) \\
\quad \operatorname{trans}\left(\mathbf{x}_{j}, d_{i}\right) \operatorname{rot}\left(\mathbf{z}_{i}, \theta_{i}\right) \operatorname{trans}\left(\mathbf{z}_{i}, r_{i}\right)
\end{array}
$$

where $\gamma_{i}, \alpha_{i}$ and $\theta_{i}$ are angles and $b_{i}, d_{i}$ and $r_{i}$ are distances. The description is extended by three parameters. The binary variable $\sigma_{i}$, is equal to one if the joint $i$ is prismatic and zero if it is rotoid. The binary variable $\mu_{i}$, is equal to one if the joint $i$ is motorized and zero if not. The variable $a(i)$ specifies the number of the body preceding the body $C_{i}$. The geometry of each kinematic chain of the device can thus be easily defined by table I.

\section{Table I}

GeOMETRICAL PARAMETERS

\begin{tabular}{|c|c|c|c|c|c|c|c|c|c|}
\hline Joint & $a(i)$ & $\mu_{i}$ & $\sigma_{i}$ & $\gamma_{i}$ & $b_{i}$ & $\alpha_{i}$ & $d_{i}$ & $\theta_{i}$ & $r_{i}$ \\
\hline \hline 1 & $a(1)$ & $\mu_{1}$ & $\sigma_{1}$ & $\gamma_{1}$ & $b_{1}$ & $\alpha_{1}$ & $d_{1}$ & $\theta_{1}$ & $r_{1}$ \\
\hline$\ldots$ & $\ldots$ & $\ldots$ & $\ldots$ & $\ldots$ & $\ldots$ & $\ldots$ & $\ldots$ & $\ldots$ & $\ldots$ \\
\hline $\mathrm{n}$ & $a(n)$ & $\mu_{n}$ & $\sigma_{n}$ & $\gamma_{n}$ & $b_{n}$ & $\alpha_{n}$ & $d_{n}$ & $\theta_{n}$ & $r_{n}$ \\
\hline
\end{tabular}

2) Elastic description: Clearly, Khalil and Kleinfinger's notation associates a rigid reference frame $R_{i}$ with each body $C_{i}$. In these frames the elastic behavior of the system is described using FEM.

Segmentation: In order to model the deformations, the most common finite element codes typically used are either linear EulerBernoulli, or Timoshenko beams if shear deformations are considered. Our study focussed principally on manipulators with slender bodies. Consequently shearing action can be neglected and the EulerBernoulli type beams can be used assuming small deformations. Thus, one can use the same segmentation as implemented in static cases presented in [32]-[34]. A body $C_{i}$ is segmented into $n_{i}$ beam elements $B_{i, j}$ and $2 n_{i}$ node elements.

The properties of the beams are summarized in the table II. The

Table II

BEAM PROPERTIES

\begin{tabular}{|c|c|}
\hline Length and Cross-sectional & $l_{i, j}$ and $S_{i, j}$ \\
\hline Density and Quadratic Moments & $\rho_{i, j}$ and $I_{x_{i, j}, I_{y_{i, j}}, I_{z_{i, j}}}$ \\
\hline Young's and Coulomb's Modulus & $E_{i, j}$ and $G_{i, j}$ \\
\hline
\end{tabular}

$(4 \times 1)$ vector of coordinates of any point $\mathbf{p}_{i, j}$ expressed within a reference frame $R_{k}$ and that belongs to $B_{i, j}$ is considered as the sum of a rigid component $\mathbf{r}_{i, j}$, the coordinate vector of the point which belongs to the ERLS, and an elastic component $\mathbf{e}_{i, j}$, which is the vector of the elastic displacement of this point (Figure 1):

$$
{ }^{k} \mathbf{p}_{i, j}={ }^{k} \mathbf{r}_{i, j}+{ }^{k} \mathbf{e}_{i, j} .
$$

Elastic displacements: A reference frame $R_{i, j}$ is associated with each beam $B_{i, j}$ and is defined as its $x$ axis $\left(O, \mathbf{x}_{i, j}\right)$ is along the main axis of the beam and its origin is the rigid position of the first node of the beam $\mathbf{r}_{i, 2 j-1}^{u}$. The vector of elastic displacements $\mathbf{e}_{i, j}$ of any point $\mathbf{p}_{i, j}$ which belongs to the beam $B_{i, j}$ can be expressed by

$$
{ }^{i, j} \mathbf{e}_{i, j}=\mathbf{N}_{i, j}\left[{ }^{i, j} \mathbf{u}_{i,(2 j-1)}{ }^{T} \quad{ }^{i, j} \mathbf{u}_{i, 2 j}{ }^{T}\right]^{T}
$$

where $\mathbf{N}_{i, j}$ is the geometrical interpolation matrix and $\mathbf{u}_{i, j}$ the elastic displacements of a node $j$ of the body $C_{i}$. It is described by the vector $\mathbf{u}_{i, j}=\left[\begin{array}{ll}d \mathbf{P}_{i, j}{ }^{T} & d \boldsymbol{\Phi}_{i, j}{ }^{T}\end{array}\right]^{T}$ where:

$$
\begin{aligned}
d \mathbf{P}_{i, j} & =\left[\begin{array}{lll}
d P_{x_{i, j}} & d P_{y_{i, j}} & d P_{z_{i, j}}
\end{array}\right]^{T} \\
d \mathbf{\Phi}_{i, j} & =\left[\begin{array}{lll}
d \Phi_{x_{i, j}} & d \Phi_{y_{i, j}} & d \Phi_{z_{i, j}}
\end{array}\right]^{T}
\end{aligned}
$$

represent the translational and angular displacements respectively. These notations are illustrated in Figure 1.

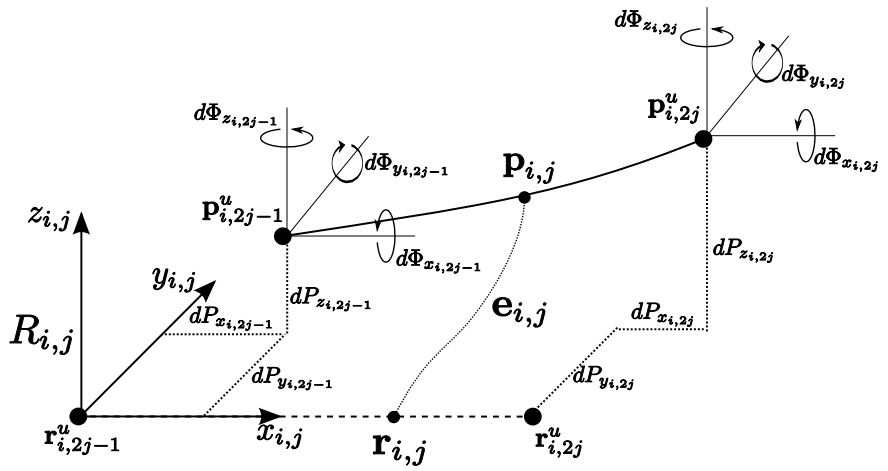

Figure 1. Notation associated with each beam $B_{i, j}$.

Assuming small elastic displacements, the following simplification can be made: $\frac{\partial d P_{y_{i, j}}}{\partial x_{i, j}} \approx d \Phi_{y_{i, j}}$ and $\frac{\partial d P_{z_{i, j}}}{\partial x_{i, j}} \approx d \Phi_{z_{i, j}}$. Consequently, the Hermite polynomials [16] are used in $\mathbf{N}_{i, j}$ to interpolate the elastic displacements due to the bending phenomenon around their axis $\mathbf{y}_{i, j}$ and $\mathbf{z}_{i, j}$. The elastic displacements due to the tractioncompression phenomenon are deduced by a linear interpolation. As a result $\mathbf{N}_{i, j}$ is defined within $R_{i, j}$ by

$$
\mathbf{N}_{i, j}=\left[\begin{array}{llll}
\mathbf{N}_{d \mathbf{P}_{i, 2 j-1}} & \mathbf{N}_{d \boldsymbol{\Phi}_{i, 2 j-1}} & \mathbf{N}_{d \mathbf{P}_{i, 2 j}} & \mathbf{N}_{d \boldsymbol{\Phi}_{i, 2 j}}
\end{array}\right]
$$

where (in the following expressions $l$ stands for $l_{i, j}$ and $\xi$ is equal $x_{i, j} / l_{i, j}$ ):

$$
\begin{array}{rl}
\mathbf{N}_{d \mathbf{P}_{i, 2 j-1}} & =\left[\begin{array}{ccc}
1-\xi & 0 & 0 \\
0 & 1-3 \xi^{2}+2 \xi^{3} & 0 \\
0 & 0 & 1-3 \xi^{2}+2 \xi^{3}
\end{array}\right] \\
0 & 0 \\
\mathbf{N}_{d \boldsymbol{\Phi}_{i, 2 j-1}} & =\left[\begin{array}{ccc}
0 & 0 & 0 \\
0 & 0 & 0 \\
0 & -l \xi+2 l \xi^{2}-l \xi^{3} & 0 \\
0 & 0 & 0
\end{array}\right] \\
\mathbf{N}_{d \mathbf{P}_{i, 2 j}} & =\left[\begin{array}{ccc}
\xi & 0 & 0 \\
0 & 3 \xi^{2}-2 \xi^{3} & 0 \\
0 & 0 & 3 \xi^{2}-2 \xi^{3} \\
0 & 0 & 0
\end{array}\right] \\
\mathbf{N}_{d \boldsymbol{\Phi}_{i, 2 j}} & =\left[\begin{array}{ccc}
0 & 0 & 0 \\
0 & 0 & -l \xi^{2}+l \xi^{3} \\
0 & l \xi^{2}-l \xi^{3} & 0 \\
0 & 0 & 0
\end{array}\right]
\end{array}
$$


In the same way, the rotation of the each beam section around its own axis $\mathbf{x}_{i, j}$ generated by the phenomenon of torsion is deduced from nodal displacements by the interpolation:

$$
{ }^{i, j} \gamma_{i, j}=\mathbf{N}_{i, j}^{t}\left[{ }^{i, j} \mathbf{u}_{i,(2 j-1)}{ }^{T} \quad{ }^{i, j} \mathbf{u}_{i, 2 j}{ }^{T}\right]^{T}
$$

where $\mathbf{N}_{i, j}^{t}$ is the $(1 \times 12)$ geometrical interpolation matrix:

$$
\mathbf{N}_{i, j}^{t}=\left[\begin{array}{lllll}
\mathbf{0}_{1,3} & 1-\xi & \mathbf{0}_{1,5} & \xi & \mathbf{0}_{1,2}
\end{array}\right] .
$$

Elastic deformations and stresses: The elastic displacements observed are caused by the elastic deformations of the beam and generate internal stresses. These deformations and stresses have to be expressed in order to evaluate the elastic potential energy of the beam. Assuming small deformations, the generalized strain vector $\boldsymbol{\epsilon}_{i, j}$ is defined within $R_{i, j}$ by

$$
{ }^{i, j} \boldsymbol{\epsilon}_{i, j}=\mathbf{B}_{i, j}\left[{ }^{i, j} \mathbf{u}_{i,(2 j-1)}{ }^{T} \quad{ }^{i, j} \mathbf{u}_{i, 2 j}{ }^{T}\right]^{T}
$$

where $\mathbf{B}_{i, j}$ is the kinematic interpolation matrix. It is obtained by derivation of the geometrical interpolation matrices (in the following expressions $x$ and $l$ stand again for $x_{i, j}$ and $l_{i, j}$ ):

$$
\mathbf{B}_{i, j}=\left[\begin{array}{llll}
\mathbf{B}_{d \mathbf{P}_{i, 2 j-1}} & \mathbf{B}_{d \boldsymbol{\Phi}_{i, 2 j-1}} & \mathbf{B}_{d \mathbf{P}_{i, 2 j}} & \mathbf{B}_{d \boldsymbol{\Phi}_{i, 2 j}}
\end{array}\right]
$$

where:

$$
\begin{aligned}
& \mathbf{B}_{d \mathbf{P}_{i, 2 j-1}}=\left[\begin{array}{ccc}
-\frac{1}{\tau} & 0 & 0 \\
0 & \frac{6}{l^{2}}-\frac{12 x}{l^{3}} & 0 \\
0 & 0 & \frac{6}{l^{2}}-\frac{12 x}{l^{3}} \\
0 & 0 & 0
\end{array}\right] \text {, } \\
& \mathbf{B}_{d \boldsymbol{\Phi}_{i, 2 j-1}}=\left[\begin{array}{ccc}
0 & 0 & 0 \\
0 & 0 & \frac{4}{l}-\frac{6 x}{l^{2}} \\
0 & -\frac{4}{l}+\frac{6 x}{l^{2}} & 0 \\
-\frac{1}{l} & 0 & 0
\end{array}\right], \\
& \mathbf{B}_{d \mathbf{P}_{i, 2 j}}=\left[\begin{array}{ccc}
\frac{1}{l} & 0 & 0 \\
0 & -\frac{6}{l^{2}}+\frac{12 x}{l^{3}} & 0 \\
0 & 0 & -\frac{6}{l^{2}}+\frac{12 x}{l^{3}} \\
0 & 0 & 0
\end{array}\right], \\
& \mathbf{B}_{d \boldsymbol{\Phi}_{i, 2 j}}=\left[\begin{array}{ccc}
0 & 0 & 0 \\
0 & 0 & \frac{2}{l}-\frac{6 x}{l^{2}} \\
0 & -\frac{2}{l}+\frac{6 x}{l^{2}} & 0 \\
\frac{1}{l} & 0 & 0
\end{array}\right] \text {. }
\end{aligned}
$$

In linear elasticity, the stress field depends on deformation and mechanical properties. By defining the $(4 \times 4)$ matrix of mechanical properties $\mathbf{E}_{i, j}$ of the beam $B_{i, j}$, the generalized stress vector $\boldsymbol{\sigma}_{i, j}$ can be written as

$$
{ }^{i, j} \boldsymbol{\sigma}_{i, j}=\mathbf{E}_{i, j}{ }^{i, j} \boldsymbol{\epsilon}_{i, j}=\mathbf{E}_{i, j} \mathbf{B}_{i, j}\left[{ }^{i, j} \mathbf{u}_{i,(2 j-1)}{ }^{T}{ }^{i, j} \mathbf{u}_{i, 2 j}{ }^{T}\right]^{T}
$$

where

$$
\mathbf{E}_{i, j}=\left[\begin{array}{cccc}
E_{i, j} S_{i, j} & 0 & 0 & 0 \\
0 & E_{i, j} I_{z_{i, j}} & 0 & 0 \\
0 & 0 & E_{i, j} I_{y_{i, j}} & 0 \\
0 & 0 & 0 & G_{i, j} I_{x_{i, j}}
\end{array}\right] .
$$

\section{3) Global expression:}

Expression in the body reference frame $R_{i}$ : Roll, pitch and yaw angles $\phi_{i, j}, \theta_{i, j}$, and $\psi_{i, j}$ are used to define the orientation of each beam $B_{i, j}$ within the reference frame $R_{i}$. The $(3 \times 3)$ rotation matrix between frames $R_{i}$ and $R_{i, j},{ }^{i} \mathbf{R}_{i, j}$, is defined by

$$
{ }^{i} \mathbf{R}_{i, j}=\operatorname{rot}\left(\mathbf{z}_{i}, \phi_{i, j}\right) \operatorname{rot}\left(\mathbf{y}_{i}, \theta_{i, j}\right) \operatorname{rot}\left(\mathbf{x}_{i}, \psi_{i, j}\right) .
$$

Then, by concatenation, the $(12 \times 12)$ rotation matrix $\mathbf{R}_{\mathbf{u}_{i, j}}$ is obtained and one can deduce the expression of $\mathbf{R}_{\mathbf{p}_{i, j}}$, the $(4 \times 4)$ transformation matrix between frames $R_{i}$ and $R_{i, j}$ :

$$
\mathbf{R}_{\mathbf{p}_{i, j}}=\left[\begin{array}{l|l}
{ }^{i} \mathbf{R}_{i, j} & { }^{i} \mathbf{r}_{i, 2 j-1}^{u}
\end{array}\right] .
$$

Assuming small displacements, the nodal displacement vector $\mathbf{u}_{i, j}$ can be rewritten in $R_{i}$ in the following way:

$$
{ }^{i} \mathbf{u}_{i, j}=\left[\begin{array}{cc}
{ }^{i} \mathbf{R}_{i, j} & \mathbf{0}_{3} \\
\mathbf{0}_{3} & { }^{i} \mathbf{R}_{i, j}
\end{array}\right]{ }^{i, j} \mathbf{u}_{i, j} .
$$

This leads to the expression of $\mathbf{p}_{i, j}$ within the reference frame $R_{i}$ :

$$
{ }^{i} \mathbf{p}_{i, j}=\mathbf{R}_{\mathbf{p}_{i, j}}{ }^{i, j} \mathbf{r}_{i, j}+\mathbf{R}_{\mathbf{p}_{i, j}} \mathbf{N}_{i, j} \mathbf{R}_{\mathbf{u}_{i, j}}\left[{ }^{i} \mathbf{u}_{i,(2 j-1)}{ }^{T}{ }^{i} \mathbf{u}_{i, 2 j}{ }^{T}\right]_{(15)}^{T} .
$$

Expression in the global reference frame $R_{0}$ : Using the ERLS description, $\mathbf{p}_{i, j}$ can be easily expressed within the global reference frame $R_{0}$ :

$$
{ }^{0} \mathbf{p}_{i, j}={ }^{0} \mathbf{T}_{i}{ }^{i} \mathbf{p}_{i, j} .
$$

Similarly, assuming small displacements, $\mathbf{u}_{i, j}$ is expressed within $R_{0}$ as:

$$
{ }^{0} \mathbf{u}_{i, j}={ }^{0} \mathbf{A}_{i}^{u i} \mathbf{u}_{i, j}
$$

where ${ }^{0} \mathbf{A}_{i}^{u}$ is a $(6 \times 6)$ rotation matrix defined by concatenation of the orientation part of ${ }^{0} \mathbf{T}_{i}$.

The concatenation of the relations obtained for each beam element leads to simple vectorial expressions to describe the kinematics of the structure studied. The $\left(4 \sum_{i=1}^{n} n_{i} \times 1\right)$ vectors of the positions $\mathbf{p}_{i, j}$ within the global reference frame $R_{0}$ is expressed by

$$
\mathbf{p}=\operatorname{Tr}+\mathbf{T N A u}
$$

where $\mathbf{r}$ is a $\left(4 \sum_{i=1}^{n} n_{i} \times 1\right)$ vector of the ERLS component expressed within each body reference frame and $\mathbf{u}$ is a $\left(12 \sum_{i=1}^{n} n_{i} \times 1\right)$ vector of the nodal coordinates expressed within $R_{0} . \mathbf{N}, \mathbf{A}$ and $\mathbf{T}$ are respectively $\left(4 \sum_{i=1}^{n} n_{i} \times 12 \sum_{i=1}^{n} n_{i}\right),\left(12 \sum_{i=1}^{n} n_{i} \times 12 \sum_{i=1}^{n} n_{i}\right)$ and $\left(4 \sum_{i=1}^{n} n_{i} \times 4 \sum_{i=1}^{n} n_{i}\right)$ matrices. They are respectively obtained by concatenating the geometric interpolation matrices $\mathbf{N}_{i, j}$ expressed in $R_{i}$ using $\mathbf{R}_{\mathbf{u}_{i, j}}$ and $\mathbf{R}_{\mathbf{p}_{i, j}}$ (15), the transfer matrices ${ }^{0} \mathbf{T}_{i}$ and the rotation matrices ${ }^{0} \mathbf{A}_{i}^{u}$. Similarly, the $\left(\sum_{i=1}^{n} n_{i} \times 1\right)$ vector of the beam section rotations $\gamma$, the $\left(4 \sum_{i=1}^{n} n_{i} \times 1\right)$ vectors of the structure generalized strain $\epsilon$ and structure generalized stresses $\sigma$, are defined by $\boldsymbol{\gamma}=\mathbf{N}^{t} \mathbf{A u}, \boldsymbol{\epsilon}=\mathbf{B A u}$ and $\boldsymbol{\sigma}=\mathbf{E B A u}$.

Then, by derivation, one can deduce the translation velocities vector $\dot{\mathrm{p}}$ and the angular velocities vector $\dot{\gamma}$ :

$$
\begin{array}{r}
\dot{\mathbf{p}}=\dot{\mathbf{T}} \mathbf{r}+\dot{\mathbf{T}} \mathbf{N A u}+\mathbf{T N} \dot{\mathbf{A} u}+\mathbf{T N A} \dot{\mathbf{u}}, \\
\dot{\gamma}=\mathbf{N}^{t} \dot{\mathbf{A}} \mathbf{u}+\mathbf{N}^{t} \mathbf{A} \dot{\mathbf{u}} .
\end{array}
$$

\section{Dynamic equilibrium expression}

Dynamic equilibrium is expressed using the Lagrange principle equations. The vector of the device DOF $\mathbf{w}$ is defined as the concatenation of the rigid and elastic DOF ( $\mathbf{q}$ and $\mathbf{u})$ :

$$
\mathbf{w}=\left[\begin{array}{ll}
\mathbf{q}^{T} & \mathbf{u}^{T}
\end{array}\right]^{T}
$$

where $\mathbf{q}=\left[\begin{array}{lll}q_{1} & \cdots & q_{n}\end{array}\right]^{T}$ is the vector of the joint values. Then, the well-known Lagrange principle is written as follows:

$$
\frac{\partial}{\partial t}\left(\frac{\partial L}{\partial \dot{\mathbf{w}}}\right)-\left(\frac{\partial L}{\partial \mathbf{w}}\right)=\mathbf{F}
$$

where $L=E_{c}-E_{p}$ is the difference between the kinetic and potential energies and $\mathbf{F}$ is the sum of non-conservative external forces. To apply this principle, we express the total energy of the kinematic chains. The resulting relations are then differentiated with respect to $\mathbf{w}, \dot{\mathbf{w}}$ and time. For this purpose, different operators are introduced. 
1) Kinetic energy: The total kinetic energy of the structure $E_{c}$ is the sum of the kinetic energy stemming from the translational and rotational movements of the beam sections. Then, using kinematic expressions ((19),(20)) the kinetic energy is derived by

$$
E_{c}=\frac{1}{2} \int_{m}\left(\dot{\mathbf{p}}^{T} \dot{\mathbf{p}}+\dot{\gamma}^{T} \mathbf{I}_{x} \dot{\gamma}\right) d m+\frac{1}{2} \sum_{k=1}^{n} \bar{\sigma}_{k} \dot{q}_{k} I_{k} \dot{q}_{k}
$$

where $\mathbf{I}_{x}$ is a $\left(\sum_{i=1}^{n} n_{i} \times \sum_{i=1}^{n} n_{i}\right)$ diagonal matrix gathering the quadratic moments $I_{x_{i, j}}$. $I_{k}$ is equal to the sum of inertia of the beams which are aligned with the joint axis and $\bar{\sigma}_{k}$ is the complement of the binary parameters defined in table I.

2) Potential energy: The potential energy of the system is the sum of the joint and body elastic potential energies and potential gravity energy. As presented in [32], the strain energy of the joints is introduced by replacing the kinematic relations which connect the elastic DOF with a joint stiffness matrix. The ERLS is modeled using Khalil and Kleinfinger's notation. Consequently, $z_{i}$ is the axis of the joint $i$ which links the body $C_{k}$ to the body $C_{i}$ where $k=a(i)$ (Table I). Thus, the joint stiffness matrix $\mathbf{K}_{l_{i}}$ expressed in its local coordinate system $R_{i}$ between the $2 n_{k}$ nodes of the body $C_{k}$ and the first node of the body $C_{i}$ is given by:

$$
{ }^{i} \mathbf{K}_{l_{i}}=\left[\begin{array}{c|c}
{ }^{i} \mathbf{K}_{l_{i}}^{u} & -{ }^{i} \mathbf{K}_{l_{i}}^{u} \\
\hline-{ }^{i} \mathbf{K}_{l_{i}}^{u} & { }^{i} \mathbf{K}_{l_{i}}^{u}
\end{array}\right]
$$

where ${ }^{i} \mathbf{K}_{l_{i}}^{u}=\operatorname{diag}\left[\begin{array}{llllll}K_{r_{i}} & K_{r_{i}} & K_{a_{i}} & K_{r r_{i}} & K_{r r_{i}} & K_{a r_{i}}\end{array}\right]$. $K_{r_{i}}, K_{a_{i}}, K_{r r_{i}}$ and $K_{a r_{i}}$ stand respectively for the radial, the axial, the radial rotational and the axial rotational stiffness of the $i^{\text {th }}$ joint. Assuming small displacements, the joint stiffness matrix can be written in the global frame $R_{0}$ as ${ }^{0} \mathbf{K}_{l_{i}}={ }^{0} \mathbf{A}_{i}^{u i} \mathbf{K}_{l_{i}}{ }^{0} \mathbf{A}_{i}^{u^{T}}$ and by concatenation of the $n$ matrices ${ }^{0} \mathbf{K}_{l_{i}}$, one can define the matrix $\mathbf{K}_{l}$. The deformation energy of flexible body is expressed as the integral of the product of the generalized strain vector $\epsilon$ and the generalized stress vector $\sigma$. Thus, the potential energy of the system is derived by

$$
E_{p}=\frac{1}{2}\left(\mathbf{u}^{T} \mathbf{K}_{l} \mathbf{u}+\sum_{i=1}^{n} \sum_{j=1}^{n_{i}} \int_{0}^{l_{i, j}}\left(\boldsymbol{\epsilon}^{T} \boldsymbol{\sigma}\right) d x_{i, j}\right)+\int_{m}\left(\mathbf{g}^{T} \mathbf{p}\right) d m
$$

where $\mathbf{g}$ represents the gravity field.

3) Operators: The implementation of the Lagrange principle requires the derivation of energies expressed previously. In order to simplify the expressions, we define the following matrix operators (26). These operators can be calculated by simple multiplications; a detailed example can be found in [12].

$$
\begin{array}{cc}
\frac{\partial \mathbf{T}}{\partial q_{k}}=\mathbf{U}_{k} & \frac{\partial \mathbf{A}}{\partial q_{k}}=\boldsymbol{\Lambda}_{k} \quad \frac{\partial \mathbf{U}_{\mathbf{k}}}{\partial q_{l}}=\mathbf{V}_{k, l} \quad \frac{\partial \boldsymbol{\Lambda}_{k}}{\partial q_{l}}=\boldsymbol{\Omega}_{k, l} \\
\dot{\mathbf{T}}=\sum_{k=1}^{n} \mathbf{U}_{k} \dot{q}_{k} & \ddot{\mathbf{T}}=\sum_{k=1}^{n}\left(\sum_{l=1}^{n} \mathbf{V}_{k, l} \dot{q}_{l}\right) \dot{q}_{k}+\sum_{k=1}^{n} \mathbf{U}_{k} \ddot{q}_{k} \\
\dot{\mathbf{A}}=\sum_{k=1}^{n} \boldsymbol{\Lambda}_{k} \dot{q}_{k} & \ddot{\mathbf{A}}=\sum_{k=1}^{n}\left(\sum_{l=1}^{n} \boldsymbol{\Omega}_{k, l} \dot{q}_{l}\right) \dot{q}_{k}+\sum_{k=1}^{n} \boldsymbol{\Lambda}_{k} \ddot{q}_{k}
\end{array}
$$

4) Dynamic equation: From the operators (26) and by differentiating the expressions (23) and (25), the terms describing the dynamic equilibrium of the kinematic chain are obtained:

$$
\begin{aligned}
& {\left[\begin{array}{cc}
\mathbf{M}_{q q} & \mathbf{M}_{q u} \\
\mathbf{M}_{q u}^{T} & \mathbf{M}_{u u}
\end{array}\right]\left[\begin{array}{c}
\ddot{\mathbf{q}} \\
\ddot{\mathbf{u}}
\end{array}\right]+\left[\begin{array}{cc}
\mathbf{0} & \mathbf{0} \\
\mathbf{0} & \mathbf{K}_{u u}
\end{array}\right]\left[\begin{array}{l}
\mathbf{q} \\
\mathbf{u}
\end{array}\right]} \\
& =\left(\mathbf{F}-\mathbf{F}_{c}-\mathbf{F}_{g}\right) .
\end{aligned}
$$

$\mathbf{M}_{q q}, \mathbf{M}_{u u}$ and $\mathbf{K}_{u u}$ describe respectively the distribution of mass on the joint DOF, the distribution of mass on the elastic DOF and the distribution of the intrinsic stiffness of the bodies and joints. In this expression, one can note the strong coupling between the joint and elastic DOF: the component of the mass matrix $\mathbf{M}_{q u}$ describes the effect of elastic DOF on the joint DOF mass expression and the coriolis and centrifugal force $\mathbf{F}_{c}$ are the sum of three components $\mathbf{F}_{r}, \mathbf{F}_{u}$ and $\mathbf{F}_{\dot{u}}$ which are respectively functions of $(\dot{\mathbf{q}}),(\dot{\mathbf{q}}, \mathbf{u})$ and $(\dot{\mathbf{q}}, \dot{\mathbf{u}})$. The gravity and external forces applied on the structure are expressed by $\mathbf{F}_{g}$ and $\mathbf{F}$. The generic expressions of these terms are detailed in appendix. Assuming deformations and displacements to be small, the second order terms related to the elastic variables are neglected.

\section{Constraint equations}

The dynamic equations (27) are formulated in terms of a set of coordinates that are not totally independent. The kinematic relations between the internal nodes can be expressed independently within each body reference frame $R_{i}$. This leads to linear equations using the Boolean matrix as demonstrated by Shabana in [35]. Concerning joint constraints: the kinematic relations must link nodes which belong to different bodies leading to non-linear equations which are functions of the joint parameters [35]. However, in our case the stiffness values of the joints are represented in (24); consequently the joint constraints are already considered within the formulation of the potential energy (25). A similar approach, called the penalty method, is presented in [36]. Hence, the constraint equations which link the independent elastic variables $\mathbf{u}_{i}$ to the dependent ones $\mathbf{u}_{d}$ can be written as $\mathbf{C}_{\mathbf{u}_{d}} \mathbf{u}_{d}+\mathbf{C}_{\mathbf{u}_{i}} \mathbf{u}_{i}=0$ where $\mathbf{C}_{\mathbf{u}_{i}}$ and $\mathbf{C}_{\mathbf{u}_{d}}$ are Boolean matrices. Then the elastic coordinates can be expressed by

$$
\mathbf{u}=\left[\begin{array}{c}
\mathbf{I} \\
-\mathbf{C}_{\mathbf{u}_{d}}^{-1} \mathbf{C}_{\mathbf{u}_{i}}
\end{array}\right] \mathbf{u}_{i}, \quad \ddot{\mathbf{u}}=\left[\begin{array}{c}
\mathbf{I} \\
-\mathbf{C}_{\mathbf{u}_{d}}^{-1} \mathbf{C}_{\mathbf{u}_{i}}
\end{array}\right] \ddot{\mathbf{u}}_{i}
$$

As regards joint coordinates, kinematic constraints have to be considered for mechanisms with closed kinematic chains and PKMs. In this case, the loop closure conditions are defined by nonlinear holonomic constraint equations. Much research is available on this subject and several formulations are proposed in [36]-[39]. In order to obtain the dynamic equations expressed in independent joint coordinates, embedding techniques can be used. In this method, the coordinates are partitioned in independent and dependent sets. From the kinematic constraint vector $\mathbf{C}(\mathbf{q}, t)=\mathbf{0}$, the application of Alembert's principle leads to

$$
\mathbf{C}_{\mathbf{q}_{i}} \delta \mathbf{q}_{i}+\mathbf{C}_{\mathbf{q}_{d}} \delta \mathbf{q}_{d}=\mathbf{0}
$$

where $\mathbf{q}_{i}$ and $\mathbf{q}_{d}$ are respectively the independent and dependent joint vectors. $\mathbf{C}_{\mathbf{q}_{i}}$ and $\mathbf{C}_{\mathbf{q}_{d}}$ are defined as the jacobian matrices of $\mathbf{C}(\mathbf{q}, t)$ relative to $\mathbf{q}_{i}$ and $\mathbf{q}_{d}$ [40]. Then, by successive derivations according to time, the joint acceleration vector can be written in terms of independent accelerations as

$$
\ddot{\mathbf{q}}=\left[\begin{array}{c}
\ddot{\mathbf{q}}_{i} \\
\ddot{\mathbf{q}}_{d}
\end{array}\right]=\left[\begin{array}{c}
\mathbf{I} \\
-\mathbf{C}_{\mathbf{q}_{d}}^{-1} \mathbf{C}_{\mathbf{q}_{i}}
\end{array}\right] \ddot{\mathbf{q}}_{i}+\left[\begin{array}{c}
\mathbf{0} \\
-\mathbf{C}_{\mathbf{q}_{d}}^{-1} \mathbf{F}_{l}
\end{array}\right]
$$

where $\mathbf{F}_{l}=-\left(\frac{\partial \mathbf{C}_{\mathbf{q}}}{\partial \mathbf{q}} \dot{\mathbf{q}}\right) \dot{\mathbf{q}}$ represents the constraint forces generated by the looping of the kinematic chains. As result, assuming that the joint and elastic coordinates are rearranged according to the partitioning of equalities (28) and (30), $\ddot{\mathbf{w}}_{i}=\left[\begin{array}{cc}\ddot{\mathbf{q}}_{i}^{T} & \ddot{\mathbf{u}}_{i}^{T}\end{array}\right]^{T}$ the set of independent coordinates is obtained by

$$
\ddot{\mathbf{w}}=\mathbf{B}_{\mathbf{d}_{i}} \ddot{\mathbf{w}}_{i}+\mathbf{F}_{l i},
$$

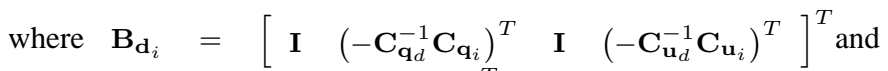
$\mathbf{F}_{l i}=\left[\begin{array}{llll}\mathbf{0} & \left(-\mathbf{C}_{\mathbf{q}_{d}}^{-1} \mathbf{F}_{l}\right)^{T} & \mathbf{0} & \mathbf{0}\end{array}\right]^{T}$. Then, the dynamic equilibrium (27) can be expressed in the base of independent parameters by

$$
\mathbf{M}_{i} \ddot{\mathbf{w}}_{i}+\mathbf{K}_{i} \mathbf{w}_{i}=\mathbf{F}_{i}
$$


where $\mathbf{M}_{i}, \mathbf{K}_{i}$ and $\mathbf{F}_{i}$ are, respectively, the mass, stiffness matrices and the force vector, defined by:

$$
\begin{aligned}
\mathbf{F}_{i} & =\mathbf{B}_{\mathbf{d}_{i}}^{T}\left(\mathbf{F}-\mathbf{F}_{c}-\mathbf{F}_{g}\right)-\mathbf{B}_{\mathbf{d}_{i}}^{T} \mathbf{M} \mathbf{F}_{l i}, \\
\mathbf{M}_{i} & =\mathbf{B}_{\mathbf{d}_{i}}^{T} \mathbf{M B}_{\mathbf{d}_{i}}, \\
\mathbf{K}_{i} & =\mathbf{B}_{\mathbf{d}_{i}}^{T} \mathbf{K B}_{\mathbf{d}_{i}} .
\end{aligned}
$$

\section{E. Identification and correlation methods}

The previous procedure allows dynamic model to be worked out from input parameters describing the mass, the damping and the stiffness properties. However, the joint stiffnesses and damping phenomenon have complex behaviors which are functions of the excitation amplitude, the frequency and the joint coordinates. The common damping models are based on parameterized rheologic laws (structural [41], the Rayleigh coefficient [17], modal damping, fractionary derivative). The non-linear behavior of joint stiffness can be described through non-parametric models (linear interpolation parameters into several configurations [41]) and parametric models (stiffness value expressed as a function of the local effort supported by the joint [42]).

Many description models can be used. The selection criteria of choice are ease of use and similarity with the experimental behavior in the workspace, for the amplitude and frequency domain of the application considered. In the pre-design stage, quantitative values are set based on the properties of the material and the designer's experience. For model correlations on a real structure and for control purposes, these values have to be accurately estimated. Then, according to research, various methods are presented involving the identification process in time [17], [43] or frequency domains [9], [41], [44], [45].

In the time domain, identification is based on the comparison of the trajectories and the efforts measured and calculated at different points of the structure. As the dynamic model does not include control and its perturbations, the actuator wrenches have to be directly measured [17]. The quality of the obtained results on trajectories used.

In the frequency domain, modal analysis is performed using specific algorithms [46] to estimate the natural frequencies and mode shapes. In the case of a slightly damped system, the conservative and damped modes present few differences. As a result, the frequencies and shapes of the theoretical normal modes obtained by solving the eigenvalues problem defined by the dynamic matrix $\mathbf{M}_{i}^{-1} \mathbf{K}_{i}$ can be directly compared to the estimated modes. This method is interesting because the stiffness and damping parameters are identified separately using a progressive approach. The first step consists of identifying the stiffness properties by minimizing frequency deviations and maximizing the Modal Assurance Criterion (MAC). This criterion evaluates the degree of linearity between the estimated and the theoretical modal vectors [47]. In step two, damping is considered to minimize the deviations between the theoretical and measured Frequency Response Functions (FRFs).

In conclusion, the level of description of the model used is a compromise between the accuracy required by the application and the cost of the experiments required to achieve the identification process.

\section{Application to A Delta like Structure}

In order to illustrate the proposed procedure, the elasto-dynamical modeling of a 3-DOF translational parallel manipulator is achieved and the potential of this structure for use in machining applications is investigated.

\section{A. System description}

The mechanical system studied here is the Surgiscope Delta-like parallel manipulator, developed by the ISIS ${ }^{1}$ company. This structure combined with a decoupled serial wrist is involved in neurosurgery to accurately move and place a microscope, a laser guiding system as well as some surgical tools (Figure 2(a)). In the following, only the positioning mechanism is considered. The mechanism described in Figure 2(b) is composed of a moving platform connected to the fixed base by three identical kinematic chains. Each kinematic chain consists of a single link-based forearm connected to a planar, four-bar parallelogram. Concerning the structure available in our laboratory, the numerical values of the main geometrical parameters are $L_{b}=$ $0.75 \mathrm{~m}$ for the length of the arms and $L_{c}=0.125 \mathrm{~m} ; L_{p}=0.95$ $\mathrm{m}$ for the width and the length of the parallelograms. The platform mass $M_{n}$ is $6.64 \mathrm{~kg}$.

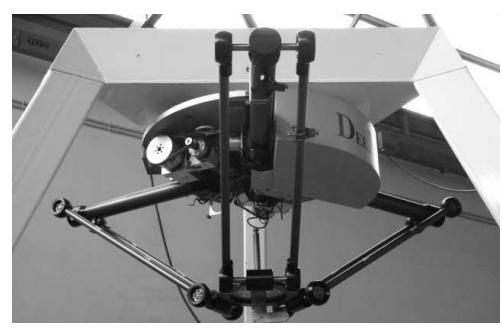

(a) Surgiscope

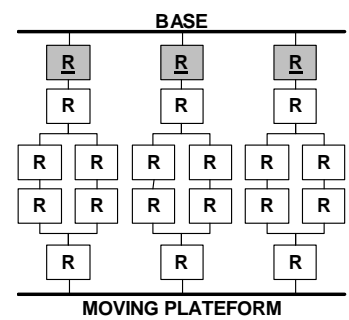

(b) Kinematics
Figure 2. Surgiscope kinematics.

\section{B. Input parameters}

The kinematic description presented in section II is implemented on each kinematic chain as indicated in Figure 3 and the associated parameter setting is summarized in Table III(a) and Table III(b). The values of these parameters are deduced from the geometrical calibration and the elastic modeling of this structure which are respectively detailed in [48] and [32]. As the structure is in a quasistatic configuration and the amplitude of the dynamic excitation is low, the effects of non-linearities are first neglected in machining applications. Then, the joint stiffness values (24) identified in [32] are used to perform the correlation on the first natural frequencies and MAC values. As the structure is axisymmetric, the elastic behavior of their joints can be described by 21 stiffness parameters. Their values were identified in [32] by minimizing the difference between the measured and modeled elastic displacement of the tool center point for a force of $-200 \mathrm{~N}$ acting along the $\mathrm{z}$ axis applied on the mobile platform. The identification was carried out with 11 measurement points distributed on the line of equation $y=-x$ in the plane $\mathrm{z}=-1478 \mathrm{~mm}$ and the identified values were experimentally checked using 11 other measurement points distributed on the line of equation $\mathrm{y}=\mathrm{x}$ in the plane $\mathrm{z}=-1326 \mathrm{~mm}$.

Concerning the damping, the models mentioned in II-E differ from each other by a variation of its frequence-dependent behavior [49]. However, for a lightly damped system at low frequencies, these models are equivalent. Consequently, we favor simplicity: structural modeling is commonly used in the frequency domain, while equivalent viscous modeling is in the time domain. We use a similar approach to the one proposed in [41] by Zhou et al. who assume that the system has structural damping proportional to the system

${ }^{1}$ ISIS: Intelligent Surgical Instrument \& Systems http://www.isisrobotics.com/ 
stiffness matrix. Then the equation of motion for free vibration of the mechanism becomes:

$$
\mathbf{M}_{i} \ddot{\mathbf{w}}_{i}+(1+j \eta) \mathbf{K}_{i} \mathbf{w}_{i}=\mathbf{0}
$$

where $\eta$ is the damping factor. This factor is identified from the measured FRFs (Figure 4(c)) by minimizing the criterion:

$$
J_{Q}=\frac{\sum_{(i, j), E, l}\left|H_{(i, j), E}^{\text {theo }}\left(\omega_{l}\right)-H_{(i, j), E}^{\text {mea }}\left(\omega_{l}\right)\right|^{2}}{\sum_{(i, j), E, l}\left|H_{(i, j), E}^{\text {mea }}\left(\omega_{l}\right)\right|^{2}}
$$

where: $H_{(i, j), E}^{\text {theo }}\left(\omega_{l}\right)$ and $H_{(i, j), E}^{\text {mea }}\left(\omega_{l}\right)$ are respectively the calculated and measured FRFs for frequency $l$ at point $(i, j)$ and for a vertical impact at the center of the platform $E$. The identified damping factor $\eta$ is 0.096 , which is high compared to standard material values (typically 0.013 for aluminium such as that used in this robot [49]) since it also includes the effects of unmodeled structural elements (e.g. joint frictions, belts, etc) not captured by the model in (33).
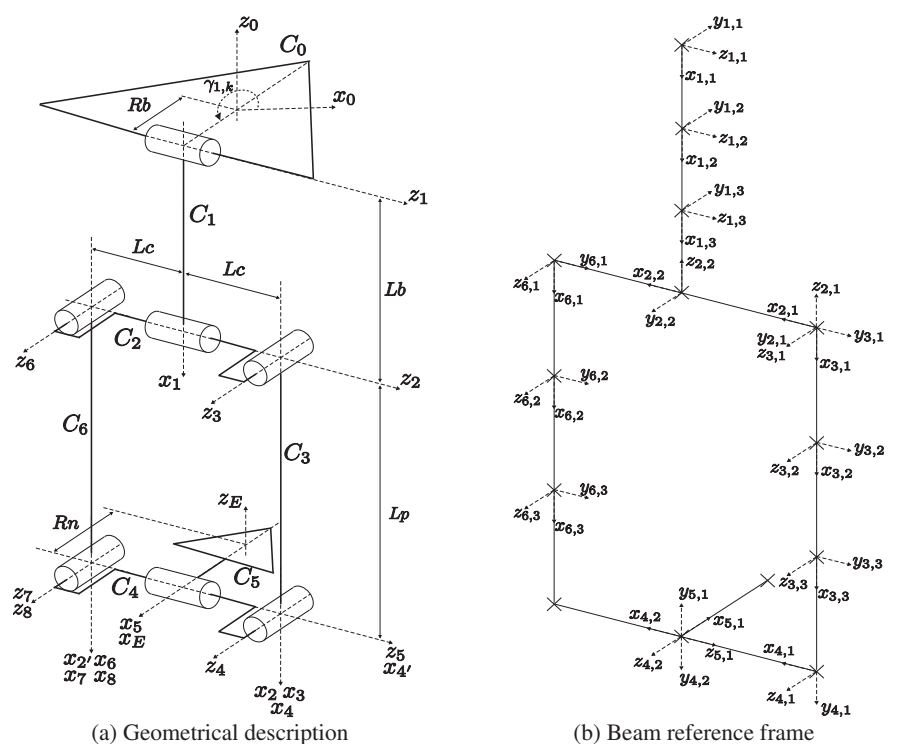

(b) Beam reference frame

Figure 3. Kinematic chain description.

\section{Measurements and correlation}

The application of experimental modal testing to the Surgiscope architecture is done through impact hammer excitation and data post-processing, conducted using SmartOffice software. The point and direction of excitation are chosen in the middle of the moving platform along the vertical axis. Piezoelectric triaxial accelerometers are used to pick up the 3 acceleration responses at 40 measured points as in the simulation. The acquisitions are performed for two structure configurations, one of which is symmetrical and the other is not (First configuration $x_{E}=y_{E}=0 \mathrm{~m}$ and $z_{E}=-1.1 \mathrm{~m}$; second configuration $x_{E}=0.19 \mathrm{~m}, y_{E}=-0.33 \mathrm{~m}$ and $z_{E}=-0.9$ $\mathrm{m})$. In order to avoid controller perturbations, the brakes are locked. Sampling parameters were specified in order to calculate the FRFs from a $2.046 \mathrm{~s}$ time window discretized with 1024 samples. The FRFs are calculated for a frequency range of 0 to $250 \mathrm{~Hz}$ at a frequency resolution of $0.49 \mathrm{~Hz}$. A force window was applied to the signal from the hammer's force transducer and exponential windows were applied to the signals from the accelerometers. Each measurement is the averaged result of three impacts. Figure 4(a), 4(b) and 4(c) show one test setting in the first configuration, the time domain measurements, and the associated FRFs, respectively.
Table III

PARAMETERS SETTING

(a) Geometrical Parameters of the $k^{t h}$ kinematic chain of the Surgiscope

\begin{tabular}{|l|l|l|l|l|l|l|l|l|l|}
\hline Joint & $a(i)$ & $\mu_{i}$ & $\sigma_{i}$ & $\gamma_{i}$ & $b_{i}$ & $\alpha_{i}$ & $d_{i}$ & $\theta_{i}$ & $r_{i}$ \\
\hline \hline 1 & 0 & 1 & 0 & $\gamma_{1, k}$ & 0 & $-\frac{\pi}{2}$ & $R b$ & $q_{1, k}$ & 0 \\
\hline 2 & 1 & 0 & 0 & 0 & 0 & 0 & $L b$ & $q_{2, k}$ & $L c$ \\
\hline 3 & 2 & 0 & 0 & 0 & 0 & $\frac{\pi}{2}$ & 0 & $q_{3, k}$ & 0 \\
\hline 4 & 3 & 0 & 0 & 0 & 0 & 0 & $L p$ & $q_{4, k}$ & 0 \\
\hline 5 & 4 & 0 & 0 & 0 & 0 & $-\frac{\pi}{2}$ & 0 & $q_{5, k}$ & $-L c$ \\
\hline 6 & 2 & 0 & 0 & 0 & $-2 L c$ & $\frac{\pi}{2}$ & 0 & $q_{6, k}$ & 0 \\
\hline 7 & 6 & 0 & 0 & 0 & 0 & 0 & $L p$ & $q_{7, k}$ & 0 \\
\hline 8 & 4 & 0 & 2 & $\frac{\pi}{2}$ & 0 & 0 & $-2 L c$ & $-\frac{\pi}{2}$ & 0 \\
\hline$E$ & 5 & 0 & 2 & 0 & 0 & $\frac{\pi}{2}$ & $-R n$ & 0 & 0 \\
\hline
\end{tabular}

\begin{tabular}{|c|c|c|c|c|c|c|c|c|c|c|c|}
\hline body & beam & $l_{i j}$ & $S_{i j}$ & $I_{y_{i j}}$ & $I_{z_{i j}}$ & $E_{i j}$ & $G_{i j}$ & $\rho_{i j}$ & $\phi_{i j}$ & $\theta_{i j}$ & $\psi_{i j}$ \\
\hline 1 & 1 & $\frac{L b}{3}$ & $S_{b}$ & $I_{y_{b}}$ & $I_{z_{b}}$ & $E_{a l}$ & $G_{a l}$ & $\rho_{a l}$ & 0 & 0 & 0 \\
\hline 1 & 2 & $\frac{L b}{3}$ & $S_{b}$ & $I_{y_{b}}$ & $I_{z_{b}}$ & $E_{a l}$ & $G_{a l}$ & $\rho_{a l}$ & 0 & 0 & 0 \\
\hline 1 & 3 & $\frac{L b}{3}$ & $S_{b}$ & $I_{y_{b}}$ & $I_{z_{b}}$ & $E_{a l}$ & $G_{a l}$ & $\rho_{a l}$ & 0 & 0 & 0 \\
\hline 2 & 1 & $L c$ & $S_{c}$ & $I_{y_{c}}$ & $I_{z_{c}}$ & $E_{a c}$ & $G_{a c}$ & $\overline{\rho_{a c}}$ & $-\frac{\pi}{2}$ & $\frac{\pi}{2}$ & $\frac{\pi}{2}$ \\
\hline 2 & 2 & $L c$ & $S_{c}$ & $I_{y_{c}}$ & $I_{z_{c}}$ & $E_{a c}$ & $G_{a c}$ & $\overline{\rho_{a c}}$ & $-\frac{\pi}{2}$ & $\frac{\pi}{2}$ & $\frac{\pi}{2}$ \\
\hline 3 & 1 & $\frac{L p}{3}$ & $S_{p}$ & $I_{y_{p}}$ & $I_{z_{p}}$ & $E_{a l}$ & $G_{a l}$ & $\rho_{a l}$ & 0 & 0 & 0 \\
\hline 3 & 2 & $\frac{L p}{3}$ & $S_{p}$ & $I_{y_{p}}$ & $I_{z_{p}}$ & $E_{a l}$ & $G_{a l}$ & $\rho_{a l}$ & 0 & 0 & 0 \\
\hline 3 & 3 & $\frac{L p}{3}$ & $S_{p}$ & $I_{y_{p}}$ & $I_{z p}$ & $E_{a l}$ & $G_{a l}$ & $\rho_{a l}$ & 0 & 0 & 0 \\
\hline 4 & 1 & $L c$ & $S_{c}$ & $I_{y_{c}}$ & $I_{z_{c}}$ & $E_{a c}$ & $G_{a c}$ & $\rho_{a c}$ & $-\frac{\pi}{2}$ & 0 & 0 \\
\hline 4 & 2 & $L c$ & $S_{c}$ & $I_{y_{c}}$ & $I_{z_{c}}$ & $E_{a c}$ & $G_{a c}$ & $\rho_{a c}$ & $-\frac{\pi}{2}$ & 0 & 0 \\
\hline 5 & 1 & $R n$ & $S_{r}$ & $I_{y_{r}}$ & $I_{z_{r}}$ & $E_{r}$ & $G_{r}$ & $\rho_{r}$ & $\pi$ & 0 & 0 \\
\hline 6 & 1 & $\frac{L p}{3}$ & $S_{p}$ & $I_{y_{p}}$ & $I_{z p}$ & $E_{a l}$ & $G_{a l}$ & $\rho_{a l}$ & 0 & 0 & 0 \\
\hline 6 & 2 & $\frac{L p}{3}$ & $S_{p}$ & $I_{y_{p}}$ & $I_{z p}$ & $E_{a l}$ & $G_{a l}$ & $\rho_{a l}$ & 0 & 0 & 0 \\
\hline 6 & 3 & $\frac{L p}{3}$ & $s_{p}$ & $I_{y_{p}}$ & $I_{z_{p}}$ & $E_{a l}$ & $G_{a l}$ & $\rho_{a l}$ & 0 & 0 & 0 \\
\hline
\end{tabular}

(b) Elastic Parameters of kinematic chain

In order to evaluate the model quality, considering all FRFs, the natural frequency deviations between test and modal analysis as well as the MAC values of the corresponding mode shapes are used. The values of natural frequencies and associated mode shapes are extracted from the experimental data using the MDOF (Multiple Degree of Freedom) estimation algorithm available on NV Solutions SmartOffice $^{2}$. This algorithm, called Polyreference Time Domain, is based on finite difference and quadrature methods [50]. The experimental and theoretical natural frequencies for the first five modes and corresponding MAC values are given in Tables IV(a) and IV(b). Similar mode shapes between theoretical and experimental approaches are found at the lowest resonances. The initial modeling provides consistent correlation for the relevant mode shapes in the frequency range from 0 to $50 \mathrm{~Hz}$. The modes obtained are due to the link elasticity (phenomena of flexion of the lower arms in Figure 5(a)) as well as the joint stiffness (structural modes on Figure 5(b)). For higher frequencies, the variations increase. Indeed the behavior of the structure is more complex and the discretization that is used by the model becomes insufficient. Concerning the damping phenomenon: the structural description whose identification is detailed in III-B fits well on the range from 0 to $100 \mathrm{~Hz}$ as shown in Figure 4(c). The criterium $J_{Q}$ defined in (34) is equal to $12.7 \%$. For higher frequencies, this description is insufficient: on the range from 0 to $250 \mathrm{~Hz}, J_{Q}$ is equal to $33.6 \%$.

\section{Discussion}

The proposed procedure implemented with static joint stiffnesses and identified structural damping as input values, presents a good correlation with the measurements obtained with the impact hammer excitation. This kind of excitation is an effective method to estimate the dynamic characteristics. However it does not characterize the nonlinearities of the structure. Thus, the obtained model is limited to applications with low amplitudes of excitation. To extend its validity

\footnotetext{
${ }^{2}$ http://www.mpihome.com/english/modaltesting.htm
} 


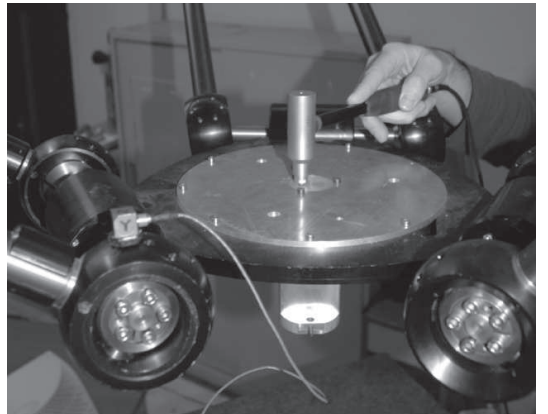

(a) Experimental Setup

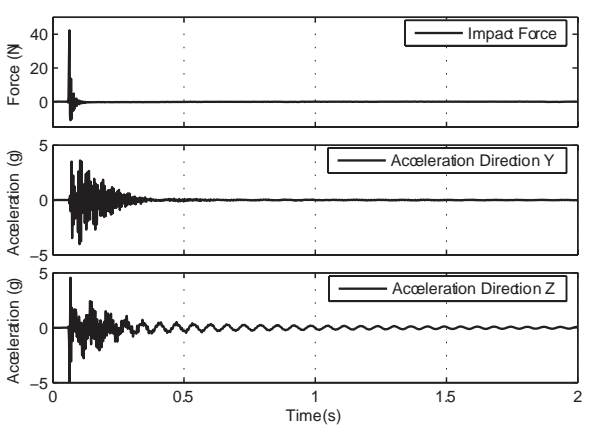

(b) Time record measurements

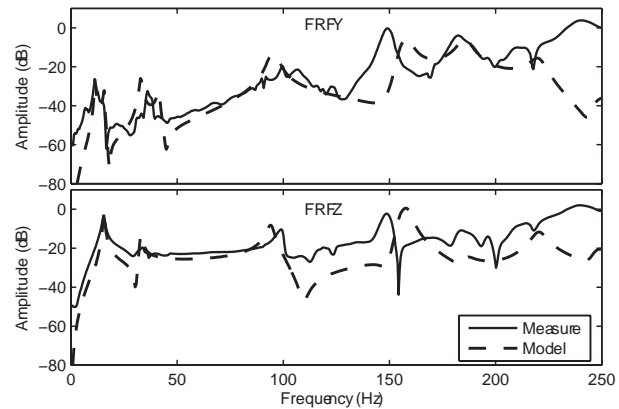

(c) Measured and Modeled FRFs

Figure 4. Experimental validation through impact hammer excitation, measurements and data post-processing.

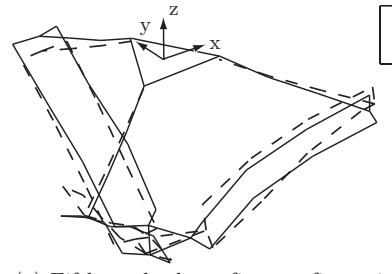

(a) Fifth mode shape first configuration

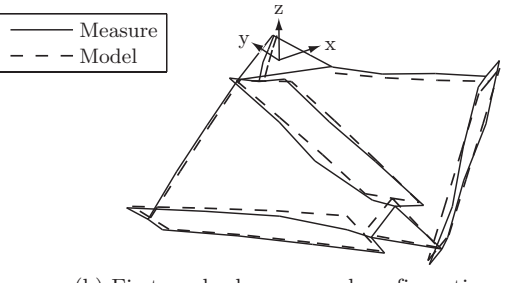

(b) First mode shape second configuration
Figure 5. Mode shape corellation.

Table IV

CORRELATION

(a) First configuration correlation

\begin{tabular}{|c|c|c|c|c|c|}
\cline { 2 - 6 } \multicolumn{1}{c|}{} & \multicolumn{6}{c|}{ Measure (Hz) } \\
\cline { 2 - 6 } \multicolumn{1}{c|}{} & Mode 1 & Mode 2 & Mode 3 & Mode 4 & Mode 5 \\
\hline Measured (Hz) & 11.8 & 15.2 & 31.2 & 36.1 & 38.8 \\
\hline Model (Hz) & 11.3 & 15.7 & 31.8 & 36.4 & 38.4 \\
\hline MAC & 0.921 & 0.964 & 0.825 & 0.673 & 0.627 \\
\hline
\end{tabular}

(b) Second configuration correlation

\begin{tabular}{|c|c|c|c|c|c|}
\cline { 2 - 6 } \multicolumn{1}{c|}{} & \multicolumn{5}{c|}{ Measured (Hz) } \\
\cline { 2 - 6 } \multicolumn{1}{c|}{} & Mode 1 & Mode 2 & Mode 3 & Mode 4 & Mode 5 \\
\hline Measure (Hz) & 12.4 & 15.4 & 26.1 & 36.2 & 42.5 \\
\hline Model (Hz) & 12.5 & 16.0 & 25.8 & 37.3 & 40.9 \\
\hline MAC & 0.956 & 0.904 & 0.833 & 0.635 & 0.641 \\
\hline
\end{tabular}

domain for many source amplitudes, a shaker excitation is more suitable. Although the duration of tests is longer, it should provide better peak to RMS level especially for low frequencies, and a good characterization of the nonlinear behavior of the structure. One of the non-linear description models detailed in II-E can be implemented and adjusted through the computational model updating method to consider the joint and damping non-linearities. However, this topic is not covered in this paper and is referred to future studies.

\section{CONCLUSION}

In this paper, a systematic procedure for the elasto-dynamic modeling of both serial and parallel three dimensional manipulators has been developed. It can be used in an industrial context for mechanical design as well as for realtime control. The identification of the input parameters according to the model application has been investigated, and an efficient identification method based on modal analysis has been detailed. The proposed procedure has been applied on a Delta like, parallel structure and successfully validated by experimental modal testing with impact hammer excitation. In order to verify the quality of the model, the natural frequency deviations between test and analysis, the MAC values of the corresponding mode shapes and the measured and computed FRFs show a consistent correlation. Current studies concern experimental investigations of damping and joint stiffness non-linearities of serial and parallel manipulators through the use of a multi-input shaker excitation.

\section{APPENDIX}

\section{MATHEMATICAL DEVELOPMENT}

The mass and stiffness matrix $\mathbf{M}_{u u}$ and $\mathbf{K}_{u u}$ are defined by

$$
\begin{aligned}
\mathbf{M}_{u u} & =\int_{m}\left(\mathbf{A}^{T} \mathbf{N}^{T} \mathbf{N} \mathbf{A}\right) d m+\int_{m}\left(\mathbf{A}^{T} \mathbf{N}^{t T} \mathbf{I}_{x} \mathbf{N}^{t} \mathbf{A}\right) d m \\
\mathbf{K}_{u u} & =\left(\sum_{i=1}^{n} \sum_{j=1}^{n_{i}} \int_{0}^{l_{i, j}}\left(\mathbf{A}^{T} \mathbf{B}^{T} \mathbf{E B A}\right) d x_{i, j}\right)+\mathbf{K}_{l} .
\end{aligned}
$$

$\mathbf{M}_{q q}$ and the vector $\mathbf{M}_{q_{k}} u$ are respectively:

$$
\begin{aligned}
& \mathbf{M}_{q_{k} q_{l}}=\int_{m}\left(\begin{array}{l}
\mathbf{r}^{T} \mathbf{U}_{k}^{T} \mathbf{U}_{l} \mathbf{r}+\mathbf{r}^{T} \mathbf{U}_{k}^{T} \mathbf{U}_{l} \mathbf{N} \mathbf{A} \mathbf{u} \\
+\mathbf{r}^{T} \mathbf{U}_{l}^{T} \mathbf{U}_{k} \mathbf{N} \mathbf{A} \mathbf{u}+\mathbf{r}^{T} \mathbf{U}_{k}^{T} \mathbf{T} \mathbf{N} \boldsymbol{\Lambda}_{l} \mathbf{u} \\
+\mathbf{r}^{T} \mathbf{U}_{l}^{T} \mathbf{T} \mathbf{N} \boldsymbol{\Lambda}_{k} \mathbf{u}
\end{array}\right) d m \\
& \begin{aligned}
\mathbf{M}_{q_{k} u}= & \int_{m}\left(\mathbf{r}^{T} \mathbf{U}_{k}^{T} \mathbf{T} \mathbf{N} \mathbf{A}+\mathbf{u}^{T}\left(\begin{array}{c}
\boldsymbol{\Lambda}_{k}^{T} \mathbf{N}^{T} \mathbf{N} \mathbf{A} \\
+\mathbf{A}^{T} \mathbf{N}^{T} \mathbf{U}_{k}^{T} \mathbf{T} \mathbf{N A}
\end{array}\right)\right) d m \\
& +\int_{m}\left(\mathbf{u}^{T} \boldsymbol{\Lambda}_{k}^{T} \mathbf{N}^{t T} \mathbf{I}_{x} \mathbf{N}^{t} \mathbf{A}\right) d m .
\end{aligned}
\end{aligned}
$$

The various coriolis, centrifugal and gravity forces are expressed by the vectors

$$
\begin{aligned}
\mathbf{F}_{r} & =\left[\begin{array}{llll}
F_{r q_{1}} & \ldots & F_{r q_{n}} & \mathbf{F}_{r u}{ }^{T}
\end{array}\right]^{T}, \\
\mathbf{F}_{u} & =\left[\begin{array}{llll}
F_{u q_{1}} & \ldots & F_{u q_{n}} & \mathbf{F}_{u u}{ }^{T}
\end{array}\right]^{T}, \\
\mathbf{F}_{\dot{u}} & =\left[\begin{array}{llll}
F_{\dot{u} q_{1}} & \ldots & F_{\dot{u} q_{n}} & \mathbf{F}_{\dot{u} u}^{T}
\end{array}\right]^{T}, \\
\mathbf{F}_{g} & =\left[\begin{array}{llll}
F_{g q_{1}} & \ldots & F_{g q_{n}} & \mathbf{F}_{g u}^{T}
\end{array}\right]^{T},
\end{aligned}
$$


where each component are defined by

$$
\begin{aligned}
& F_{r q_{k}}=\int_{m}\left(\mathbf{r}^{T} \mathbf{U}_{k}^{T} \sum\left(\sum \mathbf{V}_{i, j} \dot{q}_{j}\right) \dot{q}_{i} \mathbf{r}\right) d m, \\
& \mathbf{F}_{r u}=\int_{m}\left(\mathbf{A}^{T} \mathbf{N}^{T} \mathbf{T}^{T} \sum\left(\sum \mathbf{V}_{i, j} \dot{q}_{j}\right) \dot{q}_{i} \mathbf{r}\right) d m, \\
& F_{u q_{k}}=\int_{m}\left(\left(\begin{array}{l}
\mathbf{r}^{T} \mathbf{U}_{k}^{T} \sum\left(\sum \mathbf{V}_{i, j} \dot{q}_{j}\right) \dot{q}_{i} \mathbf{N} \mathbf{A} \\
+2 \mathbf{r}^{T} \mathbf{U}_{k}^{T} \dot{\mathbf{T}} \mathbf{N} \dot{\mathbf{A}} \\
+\mathbf{r}^{T} \sum\left(\sum \mathbf{V}_{i, j} \dot{q}_{j}\right) \dot{q}_{i}^{T} \mathbf{U}_{k} \mathbf{N} \mathbf{A} \\
+\mathbf{r}^{T} \mathbf{U}_{k}^{T} \mathbf{T} \mathbf{N} \sum\left(\sum \boldsymbol{\Omega}_{i, j} \dot{q}_{j}\right) \dot{q}_{i} \\
+\mathbf{r}^{T} \sum\left(\sum \mathbf{V}_{i, j} \dot{q}_{j}\right) \dot{q}_{i}{ }^{T} \mathbf{T} \mathbf{N} \mathbf{\Lambda}_{k}
\end{array}\right) \mathbf{u}\right) d m \\
& \mathbf{F}_{u u}=\int_{m}\left(\left(\begin{array}{l}
\mathbf{A}^{T} \mathbf{N}^{T} \mathbf{T}^{T} \sum\left(\sum \mathbf{V}_{i, j} \dot{q}_{j}\right) \dot{q}_{i} \mathbf{N A} \\
+\mathbf{A}^{T} \mathbf{N}^{T} \mathbf{T}^{T} \dot{\mathbf{T}} \mathbf{N} \dot{\mathbf{A}} \\
-\mathbf{A}^{T} \mathbf{N}^{T} \dot{\mathbf{T}}^{T} \mathbf{T} \mathbf{N} \dot{\mathbf{A}} \\
+\mathbf{A}^{T} \mathbf{N}^{T} \mathbf{N} \sum\left(\sum \boldsymbol{\Omega}_{i, j} \dot{q}_{j}\right) \dot{q}_{i}
\end{array}\right) \mathbf{u}\right) d m \\
& +\int_{m}\left(\mathbf{A}^{T} \mathbf{N}^{t T} \mathbf{I}_{x} \mathbf{N}^{t} \sum\left(\sum \boldsymbol{\Omega}_{i, j} \dot{q}_{j}\right) \dot{q}_{i} \mathbf{u}\right) d m, \\
& F_{\dot{u} q_{k}}=\int_{m}\left(2\left(\mathbf{r}^{T} \mathbf{U}_{k}^{T} \dot{\mathbf{T}} \mathbf{N} \mathbf{A}+\mathbf{r}^{T} \mathbf{U}_{k}^{T} \mathbf{T} \mathbf{N} \dot{\mathbf{A}}\right) \dot{\mathbf{u}}\right) d m, \\
& \mathbf{F}_{\dot{u} u}=\int_{m}\left(\left(\begin{array}{l}
\mathbf{A}^{T} \mathbf{N}^{T} \mathbf{T}^{T} \dot{\mathbf{T}} \mathbf{N} \mathbf{A} \\
-\mathbf{A}^{T} \mathbf{N}^{T} \dot{\mathbf{T}}^{T} \mathbf{T} \mathbf{N} \mathbf{A} \\
+2 \mathbf{A}^{T} \mathbf{N}^{T} \mathbf{N} \dot{\mathbf{A}}
\end{array}\right) \dot{\mathbf{u}}\right) d m \\
& +\int_{m}\left(2 \mathbf{A}^{T} \mathbf{N}^{t^{T}} \mathbf{I}_{x} \mathbf{N}^{t} \dot{\mathbf{A}} \dot{\mathbf{u}}\right) d m, \\
& F_{g q_{k}}=\int_{m}\left(\mathbf{g}^{T} \mathbf{U}_{k} \mathbf{r}+\mathbf{g}^{T} \mathbf{U}_{k} \mathbf{N} \mathbf{A} \mathbf{u}+\mathbf{g}^{T} \mathbf{T} \mathbf{N} \boldsymbol{\Lambda}_{k} \mathbf{u}\right) d m, \\
& \mathbf{F}_{g u}=\int_{m}\left(\mathbf{A}^{T} \mathbf{N}^{T} \mathbf{T}^{T} \mathbf{g}\right) d m
\end{aligned}
$$

\section{REFERENCES}

[1] W. J. Book, "Recursive lagrangian dynamics of flexible manipulator arms," The International J. of Robotics Research, vol. 3, no. 3, p. 87, 1984.

[2] R. F. Jacobus and M. A. Serna, "Modal analysis of a three dimensional flexible robot," Proceedings IEEE International Conference on Robotics and Automation, pp. 2962-2967, 1994.

[3] J. P. Sadler and Y. Zhijia, "A comprehensive study of modal characteristics of a cylindrical manipulator with both link and joint flexibility," Mechanism and Machine Theory, vol. 32, no. 8, pp. 941-956, 1997.

[4] W. Chen, "Dynamic modeling of multi-link flexible robotic manipulators," Computers and Structures, vol. 79, no. 2, pp. 183-195, 2001.

[5] X. Wang and J. K. Mills, "Dynamic modeling of a flexible-link planar parallel platform using a substructuring approach," Mechanism and Machine Theory, vol. 41, no. 6, pp. 671-687, 2006.

[6] S. K. Dwivedy and P. Eberhard, "Dynamic analysis of flexible manipulators, a literature review," Mechanism and Machine Theory, vol. 41, no. 7, pp. 749-777, 2006.

[7] J. Kim, F. Park, and M. Kim, "Geometric design tools for stiffness and vibration analysis of robotic mechanisms," in Proceedings IEEE International Conference on Robotics and Automation, vol. 2, 2000, pp. 1942-1947.

[8] M. Krefft and J. Hesselbach, "Elastodynamic optimization of parallel kinematics," in Proceedings IEEE International Conference on Automation Science and Engineering, 2005, pp. 357-362.

[9] X. Wang, J. Mills, and S. Guo, "Experimental identification and active control of configuration dependent linkage vibration in a planar parallel robot," IEEE Transactions on Control Systems Technology, vol. 17, no. 4, pp. 960-969, 2009.

[10] A. Shabana, "Dynamics of flexible bodies using generalized NewtonEuler equations," J. of Dynamic Systems, Measurement, and Control, vol. 112, no. 3, pp. 496-503, Sep. 1990.

[11] F. Boyer and W. Khalil, "An efficient calculation of flexible manipulator inverse dynamics," The International J. of Robotics Research, vol. 17, no. 3, p. 282, 1998.
[12] L. W. Chang and J. F. Hamilton, "The kinematics of robotic manipulators with flexible links using an equivalent rigid link system model," $J$. of Dynamic Systems, Measurement, and Control, vol. 113, p. 48, 1991.

[13] C. J. Li and T. S. Sankar, "Systematic methods for efficient modeling and dynamics computationof flexible robot manipulators," IEEE Transactions on Systems, Man and Cybernetics, vol. 23, no. 1, pp. 77-95, 1993.

[14] A. D. Luca and B. Siciliano, "Closed-form dynamic model of planar multilink lightweight robots," IEEE Transactions on Systems, Man and Cybernetics, vol. 21, no. 4, pp. 826-839, 1991.

[15] W. H. Sunada and S. Dubowsky, "On the dynamic analysis and behavior of industrial robotic manipulators with elastic members," ASME J. of Mechanism, Transmissions and Automation in Design, vol. 105, pp. 42$51,1983$.

[16] E. Bayo, "A finite-element approach to control the end-point motion of a single-link flexible robot," J. of Robotic Systems, vol. 4, no. 1, pp. 63-75, 1987.

[17] A. Gasparetto, "On the modeling of Flexible-Link planar mechanisms: Experimental validation of an accurate dynamic model," J. of Dynamic Systems, Measurement, and Control, vol. 126, p. 365, 2004.

[18] G. Piras, W. L. Cleghorn, and J. K. Mills, "Dynamic finite-element analysis of a planar high-speed, high-precision parallel manipulator with flexible links," Mechanism and Machine Theory, vol. 40, no. 7, pp. 849862, 2005.

[19] W. Khalil and M. Gautier, "Modeling of mechanical systems with lumped elasticity," Proceedings IEEE International Conference on Robotics and Automation, vol. 4, 2000.

[20] A. Shabana, "Finite element incremental approach and exact rigid body inertia," J. of Mechanical Design, vol. 118, no. 2, p. 171, 1996.

[21] J. C. Simo, "A finite strain beam formulation. the three-dimensional dynamic problem. part i," Computer Methods in Applied Mechanics and Engineering, vol. 49, no. 1, pp. 55-70, May 1985.

[22] J. C. Simo and L. Vu-Quoc, "A three-dimensional finite-strain rod model. part II: computational aspects," Computer Methods in Applied Mechanics and Engineering, vol. 58, no. 1, pp. 79-116, Oct. 1986.

[23] A. A. Shabana, "Flexible multibody dynamics: Review of past and recent developments," Multibody System Dynamics, vol. 1, no. 2, pp. 189-222, 1997.

[24] A. A. Shabana and R. Y. Yakoub, "Three dimensional absolute nodal coordinate formulation for beam elements: Theory," J. of Mechanical Design, vol. 123, no. 4, pp. 606-613, 2001.

[25] R. Y. Yakoub and A. A. Shabana, "Three dimensional absolute nodal coordinate formulation for beam elements: Implementation and applications," J. of Mechanical Design, vol. 123, no. 4, pp. 614-621, 2001.

[26] A. A. Shabana and R. Schwertassek, "Equivalence of the floating frame of reference approach and finite element formulations," International $J$. of Non-Linear Mechanics, vol. 33, no. 3, pp. 417-432, 1998.

[27] K. Dufva, J. Sopanen, and A. Mikkola, "Three-Dimensional beam element based on a Cross-Sectional coordinate system approach," Nonlinear Dynamics, vol. 43, no. 4, pp. 311-327, Mar. 2006.

[28] D. Garcia-Vallejo, J. Mayo, J. Escalona, and J. Dominguez, "Threedimensional formulation of rigid-flexible multibody systems with flexible beam elements," Multibody System Dynamics, vol. 20, no. 1, pp. $1-28,2008$.

[29] R. J. Theodore and A. Ghosal, "Comparison of the assumed modes and finite element models for flexible multilink manipulators," The International J. of Robotics Research, vol. 14, no. 2, pp. 91-111, 1995.

[30] W. Khalil and J. Kleinfinger, "A new geometric notation for open and closed-loop robots," Proceedings IEEE International Conference on Robotics and Automation, vol. 3, 1986.

[31] E. Dombre and W. Khalil, Modelisation et commande des robots. Hermes, 1988

[32] D. Deblaise, X. Hernot, and P. Maurine, "A systematic analytical method for PKM stiffness matrix calculation," in Proceedings IEEE International Conference on Robotics and Automation, 2006, pp. 42134219.

[33] M. Rognant and P. Maurine, "Elasto-Geometrical modelling of a pantographic linkage used as coordinate measuring arm for PKM applications," in Proceedings of the IFToMM 2007 World Congress, 2007.

[34] S. Marie and P. Maurine, "Elasto-geometrical modelling of closed-loop industrial robots used for machining applications," in Proceedings IEEE International Conference on Robotics and Automation, 2008, pp. 12941300.

[35] A. A. Shabana, "Constrained motion of deformable bodies," International J. for Numerical Methods in Engineering, vol. 32, no. 8, pp. 1813-1831, 1991. 
[36] E. Bayo and J. G. de Jalon, Kinematic and dynamic simulation of multibody systems: the real-time challenge. New-York: Springer-Verlag, 1993.

[37] J. Wang, C. M. Gosselin, and L. Cheng, "Modeling and simulation of robotic systems with closed kinematic chains using the virtual spring approach," Multibody System Dynamics, vol. 7, no. 2, pp. 145-170, 2002.

[38] J. Kovecses, J. Piedboeuf, and C. Lange, "Dynamics modeling and simulation of constrained robotic systems," IEEE/ASME Transactions on Mechatronics, vol. 8, no. 2, pp. 165-177, 2003.

[39] A. A. Shabana, Dynamics of Multibody Systems. Cambridge University Press, 2005.

[40] Y. Nakamura and M. Ghodoussi, "Dynamics computation of closed-link robot mechanisms with nonredundant and redundant actuators," IEEE Transactions on Robotics and Automation, vol. 5, no. 3, pp. 294-302, 1989.

[41] Z. Zhou, J. Xi, and C. K. Mechefske, "Modeling of a fully flexible 3PRS manipulator for vibration analysis," J. of Mechanical Design, vol. 128, p. 403, 2006.

[42] T. Bonnemains, H. Chanal, B. Bouzgarrou, and P. Ray, "Stiffness computation and identification of parallel kinematic machine tools," Journal of Manufacturing Science and Engineering, vol. 131, no. 4, p. 041013, 2009.

[43] J. Wu, J. Wang, and L. Wang, "Identification of dynamic parameter of a 3DOF parallel manipulator with actuation redundancy," Journal of Manufacturing Science and Engineering, vol. 130, no. 4, p. 041012, 2008.

[44] G. J. Wiens and D. S. Hardage, "Structural dynamics and system identification of parallel kinematic machines," in Proceedings of DETC/CIE 2006, Philadelphia, Pennsylvania USA, Sep. 2006.

[45] M. Cescon, I. Dressler, R. Johansson, and A. Robertsson, "Subspacebased identification of compliance dynamics of parallel kinematic manipulator," in Proceedings IEEE/ASME International Conference on Advanced Intelligent Mechatronics, 2009, pp. 1028-1033.

[46] R. R. Craig and M. A. Blair, "A generalized Multiple-Input, MultipleOutput modal parameter estimation algorithm," AIAA J., vol. 23, no. 6, pp. 931-937, 1985.

[47] R. Allemang, "The modal assurance criterion: Twenty years of use and abuse," S. V. Sound and vibration, vol. 37, no. 8, pp. 14-23, 2003.

[48] D. Deblaise and P. Maurine, "Effective geometrical calibration of a delta parallel robot used in neurosurgery," in IEEE/RSJ International Conference on Intelligent Robots and Systems, 2005, pp. 1313-1318.

[49] D. J. Inman, Engineering Vibration, Third edition. New Jersey: Prentice Hall, 2007.

[50] J. Leuridan, D. Brown, and R. Allemang, "Time domain parameter identification methods for linear modal analysis: a unifying approach," Journal of vibration, acoustics, stress, and reliability in design, vol. 108, no. 1, pp. 1-8, 1986. 